\title{
WHO Editorial
}

\section{Guang-Zhong Yang, ${ }^{1}$ Edward Kelley, ${ }^{2}$ Ara Darzi ${ }^{1}$}

\section{TECHNOLOGY FOR PATIENT SAFETY}

Modern medicine increasingly relies on new technology, yet technology can be a double-edged sword for patient safety. How to make effective use of technology and understand its potential implication on patient safety is a common thread shared across many programme areas of WHO Patient Safety. In order to identify and clarify the role and objectives of technology in improving patient safety across the world, and establish future priorities in research, education and implementation for patient safety, four international working groups were established in 2008 by WHO Patient Safety and Imperial College London to look at the following specific topics:

- introducing new technology safely;

- making technology safer in healthcare;

- information technology for patient safety;

- training and simulation technology.

Each working group includes representatives from high-, middle- and lowincome countries, drawing expertise from clinical medicine, academia, policy, healthcare management and industry. They are guided by a panel of international experts and draw on existing scientific literature, guidelines and recommended exemplars of technology for patient safety. The final outcome of these working groups resulted in four papers included in this special issue.

In the paper by Mytton et al ${ }^{1}$ on Introducing New Technology Safely, the key stages involved in the development and introduction of new technologies including clinical engineering, health technology assessment, regulation, training and surveillance are discussed. It highlights the fact that introducing new technology can be a sensitive process in healthcare settings. New risks and unforeseen dangers may be inadvertently introduced. In this regard, rigorous technology assessment to ensure systematic, transparent and unbiased

\footnotetext{
${ }^{1}$ Imperial College London, London, UK; ${ }^{2}$ WHO Patient Safety, (Geneva) 20, Avenue Appia, CH-1211 Geneva 27, SWITZERLAND.

Correspondence to Professor Guang-Zhong Yang, Institute of Global Health Innovation, Bessemer Building, Level 4, Imperial College London, Exhibition Road, London SW7 2AZ; g.z.yang@imperial.ac.uk
}

technological evaluation is essential. This should be combined with regulation and surveillance for maintaining the standards and detecting potential risks or problems effectively at an early stage. The paper also addresses some of the key factors that affect the introduction of new technology in the developing world.

Making existing technology safer in healthcare is one of the important topics covered by the working groups. The paper by Newton et $a^{2}$ addresses this topic first by advocating a safety culture of transparent, non-punitive reporting, then by introducing tangible mechanisms for enhancing patient safety through learning from failures. They have discussed the importance of early warning systems for poor device design, device failures as well as opportunities for pattern analysis for revealing unrecognised trends and hazards. In this paper, they have also highlighted the need for healthcare accreditation to ensure continuous evaluation and quality improvement, as well as human factors and intelligent redesign to minimise human errors. Specific challenges and key issues in developing countries are also addressed.

By examining the role of information technology (IT) in improving patient safety in healthcare, Huckvale et al ${ }^{3}$ report some of the main findings and recommendations of the ITworking group. The paper covers IT and patient safety both at the point of care and at the system/organisational level. Topics related to supporting care decisions, combating medication error, delivering patient-centred care and capturing adverse events are discussed. It also addresses issues concerning standards, implementation and opportunities of using IT to improve access to information by healthcare workers in developing countries.

Finally, in the paper by Aggarwal et al ${ }^{4}$, the role of training and simulation for patient safety is discussed. The article first outlines the scope and background of simulation-based learning and practice in healthcare environments, and then discusses the need of a systems-level approach towards effective simulation, in terms of both cost and final outcome. For patient safety, the use of simulation for recreating scenarios that are rarely experienced and assessing the actions of healthcare workers in challenging situa- tions is of particular interest. It is a powerful learning tool to achieve higher levels of competence and safer care.

Patient safety is a complex problem, involving interplay of cultural, technical, psychological, clinical and organisational factors. The four papers produced by the working groups are only intended to examine some, but not all, aspects of technology for patient safety. It is hoped that the general working practice can be adopted for addressing other related issues by combining expert consensus, literature review and published reports with experiential findings through close involvement of high-, middle- and low-income countries with contrastingly different healthcare provisions. In particular, educational bodies and health-service providers have been approached to provide information on the specific technology problems that developing countries face.

To introduce the general concept considered and the overall remit of these working groups, we have also included in this issue two papers by Rajesh Aggarwal et $a l^{5}$ and Oliver Mytton et $a l^{6}$, respectively, outlining key technological issues as applied to patient safety and what WHO should be doing about patient safety and technology in both developed and developing countries. All papers in this special issue have undergone systematic peer review; we are particularly grateful to all our reviewers for their time and effort in ensuring the scientific rigour and academic standards of these papers. We would also like to thank WHO (O Mytton, F Greaves, $\mathrm{V}$ Allan and $\mathrm{T}$ Fajeyisan) and $\mathrm{K}$ Kerr, R Davies, R Merrifield, R Newton, A Majeed and $\mathrm{R}$ Aggarwal from Imperial College.

We hope the papers included in this special issue will become important references for a wide range of audiences in addressing the current, as well as future challenges of technology for improving patient safety. More importantly, we hope that some of the issues highlighted, particularly for those related to information management for improved understanding of causes of errors, translating such understanding into safer technology and clinical practice, and effective training, simulation, combined with appropriate regulatory and management procedures will be adopted in routine practice throughout the developed and developing countries.

Competing interests WHO Patient Safety provided funding for and supported the Technology for Patient Workstream, specifically for the four working groups referred to in this supplement.

Provenance and peer review Not commissioned; not externally peer reviewed. 
Accepted 11 June 2010

Qual Saf Health Care 2010;19:i1-i2.

doi:10.1136/qshc.2010.044792

\section{REFERENCES}

1. Mytton OT, Velazquez A, Banken R, et al. Introducing new technology safely. Quality and Safety in Healthcare 2010;19(Suppl 2):9-14.
2. Newton R, Mytton OT, Aggarwal R, et al. Making existing technology safer in healthcare. Quality and Safety in Healthcare 2010;19(Suppl 2):15-24.

3. Huckvale C, Car J, Akiyama M, et al. Information technology for patient safety. Quality and Safety in Healthcare 2010; (Suppl 2):25-33.

4. Aggarwal R, Mytton OT, Derbrew M, et al. Training and simulation for patient safety. Quality and Safety in Healthcare 2010; (Suppl 2):34-43.
5. Aggarwal R, Mytton OT, Greaves F, Vincent C Technology as Applied to Patient Safety - an Overview. Quality and Safety in Healthcare 2010; (Suppl 2):3-8.

6. Mytton 0T, Greaves FEC, Stanton EAl, Donaldson LJ. What should WHO be doing about patient safety and technology? Quality and Safety in Healthcare 2010;(Suppl 2): $44-47$. 